\title{
EVALUATION OF CASPASE 3 AS AN INDICATOR FOR BREAST CANCER CHEMOTHERAPY RESPONSE
}

\author{
KAMAL BASRI SIREGAR ${ }^{1,2,3 *}$, TJAKRA WIBAWA MANUABA ${ }^{1}$, MUHAMMAD NAJIB DAHLAN LUBIS ${ }^{4}$, \\ ROSITA JUWITA SEMBIRING
}

\begin{abstract}
${ }^{1}$ Department of Surgery, Division of Surgical Oncology, Haji Adam Malik General Hospital Medan, Universitas Sumatera Utara, Indonesia. ${ }^{2}$ Stem Cells Program, Universitas Sumatera Utara, Indonesia. ${ }^{3}$ Nanomedicine Program, Universitas Sumatera Utara, Indonesia. ${ }^{4}$ Department of Anatomical Pathology, University of Sumatera Utara, Indonesia. ${ }^{5}$ Department of Clinical Pathology, University of Sumatera Utara, Indonesia. Email: siregarkamalbasri@gmail.com
\end{abstract}

Received: 22 March 2018, Revised and Accepted: 01 May 2018

ABSTRACT

Objectives: This study aimed to assess the difference of postchemotherapy caspase 3 level between triple negative breast cancer (TNBC) subjects with and without clinical response.

Methods: A total of 48 subjects with intraductal and 12 subjects with intralobular TNBC who were undergoing surgery at Adam Malik General Hospital were analyzed the response of neoadjuvant chemotherapy. Postsurgical breast tumor tissue was treated in pathology laboratory for caspase 3 analysis. The data were processed in SPSS 22 with significance limitation of 0.05 .

Results: Median levels of caspase 3 postchemotherapy were higher both in intraductal and intralobular TNBC subtype ( 6 vs. 4.5 and 5 vs. 3 , respectively) responsive group, while no changes detected in the group without clinical response. In statistical analysis, there was a significant difference of caspase 3 level postchemotherapy only in group with clinical response ( $\mathrm{p}=0.005$ in intraductal carcinoma and $\mathrm{p}=0.0031$ in intralobular carcinoma).

Conclusion: Postchemotherapy caspase 3 level increased significantly in TNBC, either intraductal or intralobular subtype, subjects with clinical response, but not in subjects without clinical response.

Keywords: Triple negative breast cancer, Caspase, Neoadjuvant chemotherapy.

(C) 2018 The Authors. Published by Innovare Academic Sciences Pvt Ltd. This is an open access article under the CC BY license (http://creativecommons. org/licenses/by/4. 0/) DOI: http://dx.doi.org/10.22159/ajpcr.2018.v11i8.26135

\section{INTRODUCTION}

Breast cancer had a significant increase in complex prevalence and mortality over time. In worldwide, there were 1.3 million women diagnosed with breast cancer each year and half of them resulted in mortality [1]. In Haji Adam Malik General Hospital, a referral hospital in North Sumatra Province in Indonesia, the incidence of breast cancer was increasing from 130 new cases in 2009, 344 new cases in 2010, and up to 628 cases in 2014 [2].

Breast cancer can be classified into four subclasses based on immunohistochemical examination: Luminal A, luminal B, HER2/ERBB2, and triple negative breast cancer (TNBC). TNBC accounts for $17-21 \%$ of cases of breast cancer [3]. Since this type of breast cancer did not have hormonal or targeted receptors, endocrine and target therapy had no benefit to TNBC. Therefore, chemotherapy plays an important role in the treatment of TNBC although with narrow therapeutic index [4].

Multicenter studies showed that $36 \%$ of patients receiving neoadjuvant chemotherapy with doxorubicin and cyclophosphamide showed complete clinical response and a good prognosis, especially for diseasefree survival $(p=0.001)[5,6]$. In the EORTC study, it was reported that almost $23 \%$ of patients who did not suit for breast-conserving surgery could be benefited from neoadjuvant chemotherapy [6]. However, there were also many cases of chemoresistance yielded in causing harm to patients without beneficence [7]. Therefore, there was an emerging need for a biomarker that can accurately identify the patient with chemosensitivity TNBC to avoid side effects in chemoresistance subjects [8]. This study aimed to assess the difference of postchemotherapy caspase 3 level between TNBC subjects with and without clinical response.

\section{METHODS}

This is an analytic study with cohort prospective design to analyze the difference of caspase 3 level postchemotherapy in the group with and without clinical response. In this study, we also differentiate the analysis between intraductal carcinoma (IDC) and intralobular carcinoma (ILC) subtypes. This study has been approved by the Local Ethical Committee of the Faculty of Medicine, University of Sumatera Utara.

All subjects with TNBC who were undergoing surgery in Adam Malik General Hospital from 2015 to 2017 were included in this study. The subject must agree and sign an agreement to undergo neoadjuvant combination chemotherapy of 3 series, after first being given an explanation of chemotherapy. Subjects were excluded if they had other malignancies, congenital anomalies, chronic kidney disease, and chronic liver disease.

Subjects were given neoadjuvant chemotherapy, docetaxel, and cisplatin before surgery. Chemotherapy clinical response was identified with Response Evaluation Criteria in Solid Tumors, with or without clinical response [9]. Postsurgical breast tumor tissue was treated in pathology laboratory for caspase 3 analysis. The tissues were fixated with buffer formalin and colored to be analyzed with antibody kit. The intensity of caspase 3 was interpreted by two pathologists, scaling from 0 to 6 [10].

We divided the IDC and ILC group for statistical analysis due to the different features of each subtype. The median level of caspase 3 pre- and post-chemotherapy was compared with the category of groups with and without clinical response. The data were processed in SPSS 22 with significance limitation of 0.05 using Chi-square and MannWhitney test. 


\section{RESULTS}

The characteristics of subjects in this study are shown in Table 1 . There were no differences in characteristics between IDC and ILC. As many as $50 \%$ of subjects with IDC had clinical response while $50 \%$ were not. In ILC, a majority of subjects (58.3\%) had no clinical response, while only $41.7 \%$ of subjects had a clinical response.

The median level of caspase 3 pre- and post-chemotherapy both in IDC and ILC is shown in Tables 2 and 3. Compared to prechemotherapy level, both IDC and ILC group had higher median level descriptively (Table 2). There was a significant difference of caspase 3 level after chemotherapy in IDC ( $p=0.005)$, but there was no difference shown in ILC ( $p=0.250)$. Further analysis, the significant difference of caspase 3 level after chemotherapy only existed in the subgroup with clinical response $(p=0.031)$, while no difference was found in the subgroup without clinical response $(p=0.079)$. In ILC group, although no significant difference of caspase 3 level was found in the whole group, a specific analysis in ILC subgroup with clinical response showed a significant difference of caspase 3 postchemotherapy $(\mathrm{p}=0.008)$ (Table 3$)$.

In IDC subgroup with clinical response, majority subjects (15 of 24 subjects, $62.5 \%$ ) showed higher caspase 3 level after chemotherapy. The similar description was showed in IDC subgroup without clinical response, in which majority of the subjects (13 of 24 subjects, $54.2 \%$ )

Table 1: Characteristics of subjects with TNBC IDC and ILC subtype

\begin{tabular}{llll}
\hline Characteristics & IDC $(\mathbf{n = 4 8})$ & ILC $(\mathbf{n = 1 2})$ & $\mathbf{p}$ \\
\hline Age & $46.1(1.6)$ & $51.6(3.0)$ & 0.130 \\
Menopausal status & & & \\
$\quad$ Had not menopause & 30 & 4 & 0.104 \\
$\quad$ Menopause & 18 & 8 & \\
Stage & 12 & 3 & 1.000 \\
$\quad$ IIIA & 36 & 9 & \\
IIIB & & & \\
Tumor status & $3.4(0.1)$ & $3.2(0.1)$ & 0.338 \\
T & $0.8(0.8)$ & $0.6(0.1)$ & 0.140 \\
N & $0(0.0)$ & $0(0.0)$ & \\
M & & 7 & 0.750 \\
Clinical response & 24 & 5 & \\
$\quad$ No response & 24 & & \\
Had response & &
\end{tabular}

Table 2: The difference of median level of prechemotherapy and postchemotherapy caspase 3 between IDC and ILC subtypes of TNBC

\begin{tabular}{lll}
\hline Median level of caspase $\mathbf{3}$ & IDC $(\mathbf{n = 4 8 )}$ & ILC $(\mathbf{n = 1 2})$ \\
\hline Prechemotherapy & $5(3-7)$ & $4(3-7)$ \\
Postchemotherapy & $6(2-8)$ & $5(3-8)$ \\
p* & 0.005 & 0.250 \\
\hline *Mann-Whitney. IDC: Intraductal carcinoma, ILC: Intralobular carcinoma,
\end{tabular}

TNBC: Triple negative breast cancer showed higher caspase 3 level after chemotherapy. Statistical analysis also showed no significant difference between both the groups $(\mathrm{p}=0.762)$. In ILC subgroup with clinical response, all subjects had higher caspase 3 level after chemotherapy. In ILC subgroup without clinical response, a majority also had higher caspase 3 level after chemotherapy ( 4 of 7 subjects, $57.1 \%$ ). Statistical analysis showed no significant difference between both the groups $(\mathrm{p}=0.240)$ (Table 4).

Not only IDC subgroup with or without clinical response showed no significant difference of caspase 3 after chemotherapy ( $p=0.762$ ) but also ILC subgroup with or without clinical response showed no significant difference of caspase 3 after chemotherapy $(p=0.240)$.

\section{DISCUSSION}

TNBC was a heterogeneous group of cancer, with high grade, high proliferation rate, aggressive, and bad prognosis. Although TNBC is sensitive to chemotherapy, there were reported many cases of chemoresistance of TNBC [11]. Koya et al. showed that neoadjuvant chemotherapy with doxorubicin, paclitaxel, and cyclophosphamide showed nearly $34 \%$ complete response overall and up to $57 \%$ in TNBC [12]. Other study showed that platinum regiment even showed a higher complete response in TNBC than non-TNBC type of breast cancer ( $80 \%$ vs. $51 \%$; $\mathrm{p}=0.005$ ) [13].

There are two subtypes of TNBC, IDC, and ILC [14]. In this study, 80\% of subjects had IDC and the remaining $20 \%$ had ILC tumor type. This result was similar to the majority of breast cancer, which was IDC (83\%) while almost all developed into invasive cancer [15]. The incidence and prevalence of IDC have increased since 190s, in which 5.8 per 100,000 in 1975 increased to 32.5 per 100,000 in 2004 [16]. It may be mammography, which increases the possibility of early detection and $60-70 \%$ reduction in mortality [17]. In histologic examination, IDC was deficient with the presence of pleomorphic abnormality in epithelial ductal cells with irregular chromatin distribution which were bounded by the basement membrane of the breast ducts. Further, it could be divided into comedo, non-comedo, and the presence of necrosis or not [18].

On the other hand, the ILC develops from the epithelial tissue of the mammary glandular epithelium and often invades normal tissue without involving the desmoplastic response [19]. In contrast to intraductal type breast cancer, ILC is characterized by mild thickening and induration of the breasts and may be clinically difficult to recognize and mammography [20]. In ultrasound, ILC appears as a heterogeneous hyperopic mass, with unclear boundaries and posterior acoustic shadows [21]. In molecular analysis, about $90 \%$ of ILCs have an E-cadherin protein expression deficiency, regulated by genomic alterations targeting the CDH1 gene on chromosome 16q22.1 [22].

Neoadjuvant chemotherapy response in TNBC patients was reported to vary in various studies. Gianni et al. studied the response to neoadjuvant chemotherapy in TNBC patients with $74 \%$ of patients giving a positive response [23]. Tewari et al. reported that neoadjuvant chemotherapy treatment in TNBC patients showed that $78 \%$ of patients responded positively with $64 \%$ of partial clinical responses and $14 \%$ complete clinical responses [24]. Unresponsive to chemotherapy can be caused by chemoresistance or that the tumor size was too large [25].

Table 3: Difference of median level of prechemotherapy and postchemotherapy caspase 3 between groups with and without clinical response in IDC and ILC subtypes of TNBC

\begin{tabular}{llllll}
\hline Median level of caspase 3 & \multicolumn{2}{l}{ IDC $(\mathbf{n = 4 8 )}$} & & ILC $(\mathbf{n = 1 2})$ \\
\cline { 2 - 3 } \cline { 5 - 6 } & Had clinical response & No clinical response & & Had clinical response & No clinical response \\
\hline Prechemotherapy & $4.5(3-7)$ & $5(3-7)$ & & $3(3-4)$ & $5(3-7)$ \\
Postchemotherapy & $6(2-8)$ & 0.079 & $5(5-8)$ & $6(4-7)$ \\
p* & 0.031 & 0.008 & 0.062 \\
\hline
\end{tabular}

*Mann-Whitney. IDC: Intraductal carcinoma, ILC: Intralobular carcinoma, TNBC: Triple negative breast cancer 
Table 4: Changing of caspase 3 level after chemotherapy in IDC and ILC subtypes of TNBC

\begin{tabular}{|c|c|c|c|c|}
\hline \multirow[t]{2}{*}{ Median level of caspase 3 changing } & \multicolumn{2}{|l|}{ IDC $(n=48)$} & \multicolumn{2}{|l|}{ ILC $(n=12)$} \\
\hline & Had clinical response & No clinical response & Had clinical response & No clinical response \\
\hline Lower/stable & 9 & 11 & 0 & 3 \\
\hline $\mathrm{p}^{*}$ & & 0.385 & & 0.159 \\
\hline
\end{tabular}

${ }^{*}$ Chi-square. IDC: Intraductal carcinoma, ILC: Intralobular carcinoma, TNBC: Triple negative breast cancer

As many as $50 \%$ of subjects with IDC had clinical response while $50 \%$ were not. In ILC, majority of subjects $(58.3 \%)$ had no clinical response, while only $41.7 \%$ subjected had clinical response. In this study, there are no differences between ICD and IDC. Thus, both the groups were equal to be statistically tested. Based on these results, it is generally known that the probability of receiving a chemotherapy response in TNBC patients is only about $50 \%$. With this result, consideration should be given to the cost and benefits of the chemotherapy treatment for TNBC patients later on

Caspase 3 is in the cytoplasm and is transported into the nucleus during apoptosis [26]. Caspase 3 functions in the cleavage of structural proteins, DNA repair enzymes, and activates endonucleases that cause DNA fragmentation in apoptotic mechanisms [27]. Caspase 3 as the executor or effector on the apoptotic process may play an important role in assessing the response or resistance of a cell to chemotherapy or cytotoxic substances [28].

Both IDC and ILC groups had a higher median level of caspase 3 postchemotherapy than prechemotherapy. However, in statistical analysis, only IDC had a significant difference of postchemotherapy caspase 3 level $(\mathrm{p}=0.005)$. Further analysis, the significant difference of caspase 3 level after chemotherapy only existed in the subgroup with clinical response $(p=0.031)$ in IDC and $(p=0.008)$ in ILC. This remarked that caspase 3 could effectively predict the presence of clinical response in TNBC, either in IDC or ILC. However, if both groups were analyzed statistically in another way, whether the caspase 3 level increased, decreased, or stable, there was no statistical difference ( $p=0.762$ in IDC and $p=0.240$ in ILC). Therefore, there was no significant difference of postchemotherapy caspase 3 only present in the subject with clinical response. The average increase in caspase 3 level was 1.5 in IDC group and 2 in ILC group.

These results indicated that caspase 3 that increases significantly after chemotherapy can predict clinical response in both IDC and ILC. O'Donovan et al. conducted on 103 breast tissue samples, 25 fibroadenoma tissue, and 5 normal tissues showed that caspase 3 precursor and caspase 3 active rates were higher in breast cancer tissue than in normal tissue $(\mathrm{p}=0.0188$ and $\mathrm{p}=0.0002)$ [29]. Branham et al. showed that there was a significant incidence of caspase 3 postchemotherapy [30]. dos Santos et al. also showed that caspase 3 could be a bioindicator of radiosensitivity [31]. Research shows that the apoptotic forces caused by chemotherapy agents depend on the concentrations given. In MCF-7, cells administered doxorubicin for $18 \mathrm{~h}$ showed a linear increase of caspase 3 in accordance with drug levels [32].

The activation of apoptotic pathways is the primary mechanism of cytotoxic drugs killing tumor cells. The presence of defects in apoptosis signaling contributes to tumor resistance [32]. Salako et al. demonstrated that TNBC treated with chemotherapy activates caspase $3 / 7$ and disruption of the f-actin organization, leading to apoptosis and decreased cell motility. Thus, theoretically, the better the effectiveness of chemotherapy, the higher the mechanism of apoptosis produced [28].

Several studies have reported the potential use of caspase 3 as a biomarker to predict tumor responses related to other prognostic variables (e.g., vascular invasion, lymph node metastasis, advanced stage clinical, and tumor size). This suggests that caspase 3 may be used as a potential factor for prognosis in some cancers by controlling those factors [33].

In this study, it was shown that caspase 3 increased significantly in chemotherapy, but it has not been used as a marker to distinguish chemotherapy-sensitive and resistant groups as no exact level of increment was known to be a significant cutoff point. Branham et al. showed that there is a higher probability of predicting chemoresistance or chemosensitivity before second cycles for showing $<1$ fold increase in caspase $3[30]$.

This study used a prospective cohort design. A prospective cohort study was an analytic study that began observations since the patient had not been exposed to exposure and followed until the exposure was completed. With the cohort design, it can be ensured that caspase 3 is measured at the right time. However, this study is a preliminary study and there were no previous similar studies so that the determination of the sample size is done in accordance with the considerations of researchers.

\section{CONCLUSION}

Postchemotherapy caspase 3 level increased significantly in TNBC, either intraductal or intralobulat subtypes, subjects with clinical response.

\section{CONFLICTS OF INTEREST}

This study has no conflict of interest to be declared.

\section{ACKNOWLEDGMENT}

The author would like to thank all the staff of Adam Malik General Hospital who direct or indirectly contributed to this study.

\section{AUTHOR'S CONTRIBUTIONS}

Kamal B. Siregar, as the first author, contributed to the idea, introduction, some part of results, and some part of the discussion. The second author, Manuaba TW, contributed to the methods and resulted part of this paper. The third and fourth authors, Lubis MND and Sembiring RJ, contributed to the analysis of caspase 3 and some parts of the discussion.

\section{REFERENCES}

1. Ponvinobala $\mathrm{K}, \mathrm{Kanchana} \mathrm{G}$, Rubalakshmi G. In vitro anticancer acivity of hydro-alcohol extract of leaves of Andrographis neesiana against PC-3 and MCF-7 cell lines. Int J Pharm Pharm Sci 2012;4:396-9.

2. PERABOI Cabang Medan. Laporan Kegiatan Dan Rencana Kerja Dua Tahunan Divisi Bedah Onkologi FK-USU/RSUP.H.ADAM MALIK; 2014

3. Wu J, Li S, Jia W, Su F. Response and prognosis of taxanes and anthracyclines neoadjuvant chemotherapy in patients with triplenegative breast cancer. J Cancer Res Clin Oncol 2001;137:1505-10.

4. Kirthi C, Adzal A, Reddy M, Ali SA, Yerramilli A, Sharma S. A study on the adverse effects of anticancer drugs in an oncology center of a tertiary care hospital. Int J Pharm Pharm Sci 2014;6:580-3.

5. Fisher B, Brown A, Mamounas E, Wieand S, Robidoux A, Margolese R, et al. Effect of preoperative chemotherapy on local-regional disease in wome with operable breast cancer: Findings from National Surgical Adjuvant Breast and Bowel Project B-18. J Clin Oncol 1997;15:2483-93.

6. Cleator S, Parton M, Dowsett $M$. The biology of neoadjuvant 
chemotherapy for breast cancer. Endocrine Related Cancer 2002;9:183-95.

7. Indran R, Tufo G, Pervaiz S, Brenner C. Recent advances in apoptosis, mitochondria and drug resistance in cancer cells. Biochim Biophys Acta 2011;1807:735-45.

8. Rakha E, Ellis I, Reis-Filho J. Are triple-negative and basal-like breast cancer synonymous? Clin Cancer Res 2008;14:618-9.

9. Bai-lin Z, Tong S, Zhang B, Zheng S, Ning LU. Polymorphisms of GSTP1 is associated with differences of chemotherapy response and toxicity in breast cancer. ??? 2011;124:199-204.

10. Vakkala M, Pääkkö P, Soni Y. Expression of caspases 3, 6 and 8 is increased in parallel with apoptosis and histological aggressiveness of the breast lesion. Br J Cancer 1999;81:592-9.

11. Dawood S, Broglio K, Esteva FJ. Survival among women with triple receptornegative breast cancer and brain metastases. Ann Oncol 2009;20:621-7.

12. Koya S, Li Y, McDaniel S. Safety and effectiveness of dose dense neoadjuvant chemotherapy in patients with stage II/III breast cancer. J Clin Oncol 2009;27:e11566.

13. Smith JW $2^{\text {nd }}$, McIntyre KJ, Acevedo PV. Results of a phase II openlabel, nonrandomized trial of oral satraplatin in patients with metastatic breast cancer. Breast Cancer Res Treat 2009;118:361-7.

14. West DC, McGrowder DA. Triple negative breast cancer: Therapeutic and prognostic implications. Asian Pac J Cancer Prev 2011;12:2129-33.

15. Lakhani S, Ellis I, Schnitt S, Tan P, van de Vijver M. WHO Classification of Tumours of the Breast. $4^{\text {th }}$ ed. Lyon, France: International Agency for Research on Cancer; 2012.

16. Shampoo MA, Kyle RA. Pioneers of mammography-warren and egan. Mayo Clin Proc 1997;72:32-5.

17. Rosner D, Bedwani RN, Vana J. Noninvasive breast carcinoma. Results of a national survey by the American College of Surgeons. Ann Surg 1980;192:139-47.

18. Sanders ME, Schuyler PA, Dupont WD, Page DL. The natural history of low-grade ductal carcinoma in situ of the breast in women treated by biopsy only revealed over 30 years of long-term follow-up. Cancer 2005; 103:2481-4

19. Hanagiri T, Nozoe T, Mizukami M, Ichiki Y, Sugaya M, Yasuda M, et al. Clinicopathological characteristics of invasive lobular carcinoma of the breast. Asian J Surg 2009;32:76-80.

20. Iorfida M, Maiorano E, Orvieto E, Maisonneuve P, Bottiglieri L, Rotmensz N, et al. Invasive lobular breast cancer: Subtypes and outcome. Breast Cancer Res Treat 2012;133:713-23.
21. Butler RS, Venta LA, Wiley EL, Ellis RL. Sonographic evaluation of infiltrating lobular carcinoma. AJR Am J Roentgenol 1999;172:325-30.

22. McCart RA, Kutasovic J, Lakhani S, Simpson P. Invasive lobular carcinoma of the breast: Morphology, biomarkers and 'omics. Breast Cancer Res 2015;17:12-5.

23. Gianni L, Eiermann W, Semiglazov V, Manikhas A, Lluch A, Tjulandin S, et al. Neoadjuvant chemotherapy with trastuzumab followed by adjuvant trastuzumab versus neoadjuvant chemotherapy alone, in patients with HER2-positive locally advanced breast cancer (the NOAH trial): A randomised controlled superiority trial with a parallel HER2-negative cohort. Lancet 2010;375:377-84.

24. Tewari M, Pradhan S, Singh U, Singh TB, Shukla HS. Assessment of predictive markers of response to neoadjuvant chemotherapy in breast cancer. Asian J Surg 2010;33:157-67.

25. Viale G. Characterization and clinical impact of residual disease after neoadjuvant chemotherapy. Breast 2013;22:88-91.

26. Blázquez S, Sirvent J, Olona M, Aguilar C, Pelegri A, Garcia JF, et al. Caspase-3 and Caspase-6 in ductal breast carcinoma: A descriptive study. Histol Histopathol 2006;21:1321-29.

27. Luo M, Lu Z, He S, Yuan K, Zhang Q, Sha M, et al. Nuclear entry of active caspase-3 is facilitated by its p3-recognition-based specific cleavage activity. Cell Res 2010;1:211-22.

28. Salako O, Poku R, Nkembo A, Amissah F, Ntantie E, Lamango N. Treatment of triple negative breast cancer-derived cells with polyisoprenylated cysteinyl amide inhibitors activates caspase $3 / 7$ and disrupts f-actin organization leading to apoptosis and diminished cell motility. FASEB 2016;30:1-3.

29. O'Donovan N, Crown J, Stunnel H, Hill AD, McDermott E, O'Higgins $\mathrm{N}$, et al. Caspase 3 in breast cancer. Clin Cancer Res 2003;9:738-42.

30. Branham KS, Krishna VM, Gopal SV, Raju AJ. Evaluation of caspase-3 as a possible indicator of apoptotic activity to assess the chemoresistance/sensitivity. J Pharm Res 2012;5:229-231

31. dos Santos N, Silva RF, Pinto MM, Silva EB, Tasat DR, Amaral A. Active caspase-3 expression levels as bioindicator of individual radiosensitivity. Anais Acad Brasil Ciencias 2017;89:649-59.

32. Yang XH, Sladek TL, Liu X. Reconstitution of Caspase-3 sensitizes MCF-7 breast cancer cells to doxorubicin- and etoposide-induced apoptosis. Cancer Res 2001;61:348-54.

33. Amelio MD, Cavalluci V, Cecconi F. Neuronal caspase-3 signaling: Not only cell death. Cell Death Differ 2010;17:1104-14. 